\title{
Charged Lepton Flavor Physics and Extra Dimensions.
}

\author{
E. O. Iltan * \\ Physics Department, Middle East Technical University \\ Ankara, Turkey
}

\begin{abstract}
We estimate the charged lepton electric dipole moments and the branching ratios of radiative lepton flavor violating decays in the framework of the two Higgs doublet model with the inclusion two extra dimensions. Here, we consider that the new Higgs doublet is accessible to one of the extra dimensions with a Gaussian profile and the fermions are accessible to the other extra dimension with uniform zero mode profile. We observe that the numerical values of the physical quantities studied enhance with the additional effects due to the extra dimensions and they are sensitive to the new Higgs localization.
\end{abstract}

*E-mail address: eiltan@newton.physics.metu.edu.tr 


\section{Introduction}

The fermion electric dipole moments (EDMs) provide considerable information about the CP violation since they are driven by the $\mathrm{CP}$ violating interactions. In the standard model (SM), the CP violation is carried by the complex Cabibo Kobayashi Maskawa (CKM) matrix elements in the quark sector. For the lepton sector, the possible lepton mixing matrix is the candidate for non vanishing $\mathrm{CP}$ violation. However, their negligibly small theoretical values stimulate one to search new models beyond the SM and one can obtain relatively greater EDMs with the extension of the particle spectrum, such as multi Higgs doublet models (MHDM), supersymmetric model (SUSY), [1,..., etc.. Among fermion EDMs, the charged lepton EDMs are worthwhile to study since they are clean theoretically. In the literature, there exist various experimental and theoretical work on the charged lepton EDMs. The experimental results for electron, muon and tau read $d_{e}=(1.8 \pm 1.2 \pm 1.0) \times 10^{-27} e \mathrm{~cm}[2], d_{\mu}=(3.7 \pm 3.4) \times 10^{-19} e \mathrm{~cm}$ [3] and $d_{\tau}=3.1 \times 10^{-16} \mathrm{ecm}$ [4], respectively. On the other hand, theoretically, the charged lepton EDMs have been predicted extensively [5]-[10]. They have been analyzed in the framework of the seesaw model in [5]. In [6], the EDMs of the charged leptons were estimated in the $2 \mathrm{HDM}$ and $d_{e}$ has been predicted at the order of the magnitude of $10^{-32} e-\mathrm{cm}$. The work [7] was devoted to the charged lepton EDMs in the framework of the SM with the inclusion of non-commutative geometry and, in [8], the effects of non-universal extra dimensions on these quantities in the two Higgs doublet model were studied . Recently, the charged lepton EDMs have been estimated in the split fermion scenario [9] and the effect of the localization of the new Higgs doublet in the split fermion scenario has been analyzed in [10]. In these theoretical works, some of the possible models and the additional effects due to the extension of the space-time have been studied to check the possible enhancement in the numerical values of the EDMs. In addition to the charged lepton EDMs the lepton flavor violating (LFV) interactions are rich from the theoretical point of view since they also exist at least at the loop level and make it possible to search the free parameters of the models used. In the SM, their branching ratios (BRs) are much below the experimental limits since their existence depends on the neutrino mixing with non zero neutrino masses and, therefore, it is worthwhile to study them in the models beyond the SM. The improvement of the experimental measurements of the LFV processes make it possible to understand the new physics effects more accurately. One of the candidate model beyond the SM is the 2HDM, where the LFV interactions are induced by the internal neutral Higgs bosons $h^{0}$ and $A^{0}$. The $\mu \rightarrow e \gamma$ and $\tau \rightarrow \mu \gamma$ are the examples of LFV interactions and the current limits for their BRs are $1.2 \times 10^{-11}\left[11\right.$ and $3.9 \times 10^{-7}[12$, respectively. A new 
experiment at PSI has been described and aimed to reach to a sensitivity of $B R \sim 10^{-14}$ for $\mu \rightarrow$ er decay [13] and, at present, the experiment (PSI-R-99-05 Experiment) is still running in the MEG [14]. For $\tau \rightarrow \mu \gamma$ decay an upper limit of $B R=9.0$ (6.8) $10^{-8}$ at $90 \%$ CL has been obtained [15] ([16]), which is an improvement almost by one order of magnitude with respect to previous one. Besides the experimental studies, there is an extensive work on the radiative LFV decays in the literature from the theoretical point of view [17]-[32]. They are analyzed in the supersymmetric models [17]-[23], [32], in a model independent way [24], in the 2HDM [24, 25, 26, 27, 28, 29, 30, 31].

This work is devoted to the prediction of the charged lepton EDMs and the BRs of the LFV processes $\mu \rightarrow e \gamma, \tau \rightarrow e \gamma$ and $\tau \rightarrow \mu \gamma$ in the 2HDM. In this model the CP violating nature of the charged lepton EDMs are carried by the complex Yukawa couplings connected to the new Higgs-lepton-lepton vertices and they are induced by the internal new neutral Higgs bosons $h^{0}$ and $A^{0}$. Similarly, the lepton flavor violation depends on these vertices and the necessary loop diagrams contain internal neutral Higgs bosons $h^{0}$ and $A^{0}$. Furthermore, we extend the spacetime with the additional two spatial dimensions and we consider that the new Higgs doublet feels one of the extra dimensions (the sixth one) with a Gaussian profile, and the fermions feel the other extra dimension (the fifth one), with uniform zero mode profile.

The extra dimension scenario is a candidate as a possible solution to the hierarchy problem of the SM. In the literature [33]-[75], the effects of extra dimensions on various phenomena have been studied extensively. In the extra dimension scenarios the compactification of extra dimension to a circle $S^{1}$ with radius $R$ results in appearing new particles, namely Kaluza-Klein $(\mathrm{KK})$ modes in the theory. In the case that all the fields feel the extra dimensions, so called universal extra dimensions (UED), the extra dimensional momentum, therefore the KK number at each vertex, is conserved. If the extra dimensions are not felt by some fields in the theory, such type is called non-universal extra dimensions where there is no restriction to conserve the KK number at each vertex. This leads to the possibility of the tree level interaction of KK modes with the ordinary particles. In another scenario, so called the split fermion scenario, the fermions are assumed to locate at different points in the extra dimension with Gaussian profiles and the hierarchy of fermion masses can be obtained from the overlaps of fermion wave functions [76]-[91].

In the present work, we consider two extra dimensions and assume that the new Higgs doublet is accessible to one of the extra dimensions with a Gaussian profile around origin and also around another point near to the origin. In addition to this, we take the fermions 
are accessible to the other extra dimension with uniform zero mode profile. Here the extra dimensions are compactified to the orbifold $S^{1} / Z^{2} \times S^{1}$ so that the chiral structure of four dimensional fermions are ensured. The localization of Higgs bosons in the extra dimension has been considered previously. The idea of the localization of the SM Higgs, using the localizer field, has been studied in [92]. [10] was devoted to the localized new Higgs scalars in the extra dimension where the localization is measured by the strength of the small coupling of the localizer field to the new Higgs scalar. In [30], the BRs of the radiative LFV decays in the split fermion scenario, with the assumption that the new Higgs doublet is restricted to the 4D brane or to a part of the bulk in one and two extra dimensions, in the framework of the 2HDM has been studied. In [31, 93] the new Higgs doublet localization effects on radiative LFV decays and LFV Z boson decays have been estimated.

Our analysis shows that the inclusion of lepton KK modes due to the fifth dimension results in an enhancement in the charged lepton EDMs and the BRs of LFV $l_{i} \rightarrow l_{j} \gamma$ decays. Furthermore, we observe that these physical quantities are strongly sensitive to the location of the new Higgs doublet Gaussian profiles in the sixth dimension.

The paper is organized as follows: In Section 2, we present EDMs of the charged leptons and the BRs of the radiative LFV decays in the $2 \mathrm{HDM}$ with the inclusion of two spatial extra dimensions. Section 3 is devoted to discussion and our conclusions.

\section{Charged Lepton Flavor Physics in the two Higgs dou- blet model where the leptons and the new Higgs dou- blet feel different extra dimensions.}

\subsection{Electric dipole moments of charged leptons}

The fermion EDM carries a valuable information about the existence of the CP violation since it emerges from the CP violating fermion-fermion-photon interaction. The possible source of CP the violation is the complex CKM matrix (lepton mixing matrix) elements for quarks (for leptons), in the framework of the SM. However, their estimated numerical values are extremely small and this makes it interesting to investigate new complex phases by considering the physics beyond the SM. The extension of the Higgs sector may bring additional complex phases with the assumption that the flavor changing neutral currents (FCNC) are permitted at tree level with new complex Yukawa couplings. The 2HDM is one of the candidate to switch on the additional CP phase to enhance the amount of the possible CP violation. On the other hand, 
the addition of the spatial extra dimensions bring new contributions which are sensitive to the compactification scale $1 / R$ where $R$ is the radius of the compactification. In the present work, we consider two additional dimensions and assume that the new Higgs doublet feels one of the extra dimensions with a Gaussian profile, and the fermions accessible to the other one.

The Yukawa Lagrangian responsible for the lepton EDM in a two extra dimensions, respecting the considered scenario, reads:

$$
\mathcal{L}_{Y}=\left.\left.\left.\xi_{6 i j}^{E} \bar{l}_{i L}\right|_{z=0} \phi_{2}\right|_{y=0} E_{j R}\right|_{z=0}+\text { h.c. }
$$

where $L$ and $R$ denote chiral projections $L(R)=1 / 2\left(1 \mp \gamma_{5}\right)$. Here $l_{i L}\left(E_{j R}\right)$, with family indices $i, j$, are the lepton doublets (singlets), $\phi_{2}$ is the new Higgs doublet.We choose the Higgs doublets $\phi_{1}$ and $\phi_{2}$ as

$$
\phi_{1}=\frac{1}{\sqrt{2}}\left[\left(\begin{array}{c}
0 \\
v+H^{0}
\end{array}\right)+\left(\begin{array}{c}
\sqrt{2} \chi^{+} \\
i \chi^{0}
\end{array}\right)\right] ; \phi_{2}=\frac{1}{\sqrt{2}}\left(\begin{array}{c}
\sqrt{2} H^{+} \\
H_{1}+i H_{2}
\end{array}\right)
$$

with the vacuum expectation values,

$$
<\phi_{1}>=\frac{1}{\sqrt{2}}\left(\begin{array}{l}
0 \\
v
\end{array}\right) ;<\phi_{2}>=0,
$$

and collect SM (new) particles in the first (second) doublet. Notice that $H_{1}$ and $H_{2}$ are the mass eigenstates $h^{0}$ and $A^{0}$ respectively since no mixing occurs between two CP-even neutral bosons $H^{0}$ and $h^{0}$ at tree level, in our case. Here, we assume that the new Higgs scalars $\left(S=h^{0}, A^{0}\right)$ are localized in the extra dimension at the point $z_{H}, z_{H}=\alpha \sigma$ with Gaussian profiles,

$$
S(x, z)=A_{H} e^{-\beta\left(z-z_{H}\right)^{2}} S(x)
$$

by an unknown mechanism 1 with the normalization constant

$$
A_{H}=\frac{2(\beta)^{1 / 4}}{\left.(2 \pi)^{1 / 4} \sqrt{\operatorname{Erf}\left[\sqrt{2 \beta}\left(\pi R+z_{H}\right)\right]+\operatorname{Erf}\left[\sqrt{2 \beta}\left(\pi R-z_{H}\right)\right.}\right]} .
$$

Here the parameter $\beta=1 / \sigma^{2}$ regulates the amount of localization, where $\sigma, \sigma=\rho R$, is the Gaussian width of $S(x, z)$ in the extra dimension. The function $\operatorname{Er} f[z]$ is the error function, which is defined as

$$
\operatorname{Erf}[z]=\frac{2}{\sqrt{\pi}} \int_{0}^{z} e^{-t^{2}} d t
$$

\footnotetext{
${ }^{1}$ We consider the zero mode Higgs scalars and we do not take into account the possible KK modes of Higgs scalars since the mechanism for the localization is unknown and we expect that the those contributions are small due to their heavy masses.
} 
On the other hand, the five dimensional lepton doublets and singlets have both chiralities and the four dimensional Lagrangian is constructed by expanding these fields into their KK modes. Besides, the fifth extra dimension denoted by $y$ is compactified on an orbifold $S^{1} / Z_{2}$ with radius $R$. The KK decompositions of the lepton fields read

$$
\begin{aligned}
l_{i}(x, y) & =\frac{1}{\sqrt{2 \pi R}}\left\{l_{i L}^{(0)}(x)+\sqrt{2} \sum_{n=1}^{\infty}\left[l_{i L}^{(n)}(x) \cos (n y / R)+l_{i R}^{(n)}(x) \sin (n y / R)\right]\right\} \\
E_{i}(x, y) & =\frac{1}{\sqrt{2 \pi R}}\left\{E_{i R}^{(0)}(x)+\sqrt{2} \sum_{n=1}^{\infty}\left[E_{i R}^{(n)}(x) \cos (n y / R)+E_{i L}^{(n)}(x) \sin (n y / R)\right]\right\}
\end{aligned}
$$

where, $l_{i L}^{(0)}(x)$ and $E_{i R}^{(0)}(x)$ are the four dimensional lepton doublets and lepton singlets respectively.

Now, we present the effective EDM interaction for a charged lepton $l$ and it reads

$$
\mathcal{L}_{E D M}=i d_{l} \bar{l} \gamma_{5} \sigma^{\mu \nu} l F_{\mu \nu}
$$

where $F_{\mu \nu}$ is the electromagnetic field tensor, ' $d_{l}$ ' is EDM of the charged lepton $l$ and it is a real number by hermiticity. In Fig. 1 we present the 1-loop diagrams which contribute to the EDMs of leptons with the help of the complex Yukawa couplings. Here, we assume that there is no CKM type lepton mixing matrix and, therefore, only the neutral Higgs part gives a contribution to their EDMs. The complex Yukawa couplings of the new Higgs doublet to the leptons play the main role in the determination of lepton EDM and they are modified with the reduction of the extra dimensions. To obtain the lepton-lepton-Higgs interaction coupling in four dimensions we need to integrate the combination $\bar{l}_{i L(R)}^{(0(n))}(x, y) S(x, z) l_{j R(l)}^{(n(0))}(x, y)$, appearing in the part of the Lagrangian (eq. (10)), over the fifth and sixth dimensions. Using the KK basis for lepton fields (see eq. (17)), we get

$$
\int_{-\pi R}^{\pi R} d z \int_{-\pi R}^{\pi R} d y \delta(z) \delta(y) \bar{l}_{i L(R)}^{(0(n))}(x, y) S(x, z) l_{j R(L)}^{(n(0))}(x, y)=V_{n} \bar{l}_{i L(R)}^{(0(n))}(x) S(x) l_{j R(L)}^{(n(0))}(x)
$$

where the factor $V_{n}$ reads

$$
V_{n}=\frac{A_{H}}{2 \pi R}
$$

and the function $A_{H}$ is defined in eq. (5) . Here, the fields $l_{i L}^{(n(0))}, l_{i R}^{(n(0))}$ are four dimensional left and right handed zero (n) mode lepton fields. Here we define the Yukawa couplings in four dimensions as

$$
\xi_{i j}^{E}=V_{n} \xi_{6 i j}^{E}
$$


where $\xi_{6 i j}^{E}$ are Yukawa couplings in six dimensions (see eq. (11) $) 2$ Notice that we consider the compactification of two extra dimensions on $\left(S^{1} / Z_{2} \times S^{1}\right)$.

Finally, the EDMs $d_{l}$ of charged leptons $(l=e, \mu, \tau)$ can be calculated as a sum of contributions coming from neutral Higgs bosons $h_{0}$ and $A_{0}$,

$$
\begin{aligned}
d_{l} & =-\frac{i G_{F}}{\sqrt{2}} \frac{e}{32 \pi^{2}} Q_{\tau} c_{H}\left(\left(\bar{\xi}_{N, l \tau}^{D *}\right)^{2}-\left(\bar{\xi}_{N, \tau l}^{D}\right)^{2}\right)\left(\frac{1}{m_{\tau}}\left(F_{1}\left(y_{h_{0}}\right)-F_{1}\left(y_{A_{0}}\right)\right)\right. \\
& \left.+2 \sum_{n=1}^{\infty} \frac{1}{\sqrt{m_{\tau}^{2}+m_{n}^{2}}}\left(F_{1}\left(y_{n, h_{0}}\right)-F_{1}\left(y_{n, A_{0}}\right)\right)\right)
\end{aligned}
$$

for $l=e, \mu$ and

$$
\begin{aligned}
d_{\tau} & =-\frac{i G_{F}}{\sqrt{2}} \frac{e}{32 \pi^{2}} Q_{\tau} c_{H}\left(\left(\bar{\xi}_{N, \tau \tau}^{D *}\right)^{2}-\left(\bar{\xi}_{N, \tau \tau}^{D}\right)^{2}\right)\left(\frac{1}{m_{\tau}}\left(F_{2}\left(r_{h_{0}}\right)-F_{2}\left(r_{A_{0}}\right)\right)\right. \\
& \left.+2 \sum_{n=1}^{\infty} \frac{1}{\sqrt{m_{\tau}^{2}+m_{n}^{2}}}\left(F_{1}\left(y_{n, h_{0}}\right)-F_{1}\left(y_{n, A_{0}}\right)\right)\right)
\end{aligned}
$$

where

$$
c_{H}=e^{\frac{-2 z_{H}^{2}}{\sigma^{2}}},
$$

for the case that the new Higgs scalars $S$ are localized around the point $z_{H}$ different than origin. If the new Higgs localization is around the origin $c_{H}$ reaches one. The functions $F_{1}(w), F_{2}(w)$ read

$$
\begin{aligned}
& F_{1}(w)=\frac{w\left(3-4 w+w^{2}+2 \ln w\right)}{(-1+w)^{3}} \\
& F_{2}(w)=w \ln w+\frac{2(-2+w) w \ln \frac{1}{2}(\sqrt{w}-\sqrt{w-4})}{\sqrt{w(w-4)}}
\end{aligned}
$$

with $y_{n, S}=\frac{m_{\tau}^{2}+m_{n}^{2}}{m_{S}^{2}}, m_{n}=\frac{n}{R}, y_{S}=y_{0, S}, r_{S}=\frac{1}{y_{S}}$ and $Q_{\tau}$ is charge of $\tau$ lepton. In eq. (12) and (13) we take into account only internal $\tau$-lepton contribution respecting our assumption that the Yukawa couplings $\bar{\xi}_{N, i j}^{E}, i, j=e, \mu$, are small compared to $\bar{\xi}_{N, \tau i}^{E} i=e, \mu, \tau$ due to the possible proportionality of the Yukawa couplings to the masses of leptons in the vertices [94]. Here we used the parametrization

$$
\bar{\xi}_{N, \tau l}^{E}=\left|\bar{\xi}_{N, \tau l}^{E}\right| e^{i \theta_{l}}
$$

Therefore, the Yukawa factors in eqs. (12), (13) can be written as

$$
\left(\left(\bar{\xi}_{N, l \tau}^{D *}\right)^{2}-\left(\bar{\xi}_{N, \tau l}^{D}\right)^{2}\right)=-2 i \sin 2 \theta_{l}\left|\bar{\xi}_{N, \tau l}^{D}\right|^{2},
$$

\footnotetext{
${ }^{2}$ In the following we use the dimensionful complex coupling $\bar{\xi}_{N}^{E}$ with the definition $\xi_{N, i j}^{E}=\sqrt{\frac{4 G_{F}}{\sqrt{2}}} \bar{\xi}_{N, i j}^{E}$ where $\mathrm{N}$ denotes the word "neutral".
} 
where $l=e, \mu, \tau$ and $\theta_{l}$ is the $\mathrm{CP}$ violating parameter which is the source of the lepton EDM. Notice that, we make our calculations in arbitrary photon four momentum square $q^{2}$ and take $q^{2}=0$ at the end.

\section{$2.2 \quad$ The radiative $\mathrm{LFV}$ decays}

LFV $l_{1} \rightarrow l_{2} \gamma$ decays exist at loop level in the SM and the numerical values of their BRs are far from the experimental estimates. Therefore, one goes the models beyond where the particle spectrum is extended and the additional contributions result in an enhancement in the numerical values of the physical parameters. Due to the extended Higgs sector, the version of the 2HDM, permitting the existence of the FCNCs at tree level, is one of the candidate to obtain relatively large BRs of the decays under consideration. Furthermore, we take into account the effects of two spatial extra dimensions which causes to enhance the BRs due to the fact that the particle spectrum is further extended after the compactification. Here, we consider the effects of the additional Higgs sector with the assumption that the new Higgs scalar zero modes are localized in one of the extra dimension with Gaussian profiles by an unknown mechanism, on the other hand, the zero modes of charged leptons have uniform profile in the other extra dimension. The Yukawa Lagrangian responsible for the LFV interactions in two extra dimensions are given in eq. (11).

Now, we will present the decay widths of the processes $\mu \rightarrow e \gamma, \tau \rightarrow e \gamma$ and $\tau \rightarrow \mu \gamma$. Since they appear at least at one loop level in the 2HDM (see Fig. 1) there exist the logarithmic divergences in the calculations. These divergences can be eliminated by using the on-shell renormalization scheme 3 . The decay width $\Gamma$ for the $l_{1} \rightarrow l_{2} \gamma$ decay reads

$$
\Gamma\left(l_{1} \rightarrow l_{2} \gamma\right)=c_{1}\left(\left|A_{1}\right|^{2}+\left|A_{2}\right|^{2}\right)
$$

for $l_{1}\left(l_{2}\right)=\tau ; \mu(\mu$ or $e ; e)$. Here $c_{1}=\frac{G_{F}^{2} \alpha_{e m} m_{l_{1}}^{3}}{32 \pi^{4}}, A_{1}\left(A_{2}\right)$ is the left (right) chiral amplitude and taking only $\tau$ lepton for the internal line, they read

$$
\begin{aligned}
A_{1} & =Q_{\tau} \frac{1}{48 m_{\tau}^{2}}\left\{6 m _ { \tau } \overline { \xi } _ { N , \tau l _ { 2 } } ^ { E * } \overline { \xi } _ { N , l _ { 1 } \tau } ^ { E * } c _ { H } \left(\left(F_{1}\left(y_{h^{0}}\right)-F_{1}\left(y_{A^{0}}\right)\right)\right.\right. \\
& \left.+2 \sum_{n=1}^{\infty} \frac{m_{\tau}}{\sqrt{m_{\tau}^{2}+m_{n}^{2}}}\left(F_{1}\left(y_{n, h^{0}}\right)-F_{1}\left(y_{n, A^{0}}\right)\right)\right)+m_{l_{1}} \bar{\xi}_{N, \tau l_{2}}^{E *} \bar{\xi}_{N, \tau l_{1}}^{E} c_{H}\left(\left(G\left(y_{h^{0}}\right)+G\left(y_{A^{0}}\right)\right)\right.
\end{aligned}
$$

\footnotetext{
${ }^{3}$ In this scheme, the self energy diagrams for on-shell leptons vanish since they can be written as $\sum(p)=$ $\left(\hat{p}-m_{l_{1}}\right) \bar{\sum}(p)\left(\hat{p}-m_{l_{2}}\right)$, however, the vertex diagrams (see Fig 1) give non-zero contribution. In this case, the divergences can be eliminated by introducing a counter term $V_{\mu}^{C}$ with the relation $V_{\mu}^{R e n}=V_{\mu}^{0}+V_{\mu}^{C}$, where $V_{\mu}^{R e n}\left(V_{\mu}^{0}\right)$ is the renormalized (bare) vertex and by using the gauge invariance: $k^{\mu} V_{\mu}^{R e n}=0$. Here, $k^{\mu}$ is the four momentum vector of the outgoing photon.
} 


$$
\begin{aligned}
& \left.\left.+2 \sum_{n=1}^{\infty} \frac{m_{\tau}^{2}}{m_{\tau}^{2}+m_{n}^{2}}\left(G\left(y_{n, h^{0}}\right)+G\left(y_{n, A^{0}}\right)\right)\right)\right\} \\
A_{2} & =Q_{\tau} \frac{1}{48 m_{\tau}^{2}}\left\{6 m _ { \tau } \overline { \xi } _ { N , l _ { 2 } \tau } ^ { E } \overline { \xi } _ { N , \tau l _ { 1 } } ^ { E } c _ { H } \left(\left(F_{1}\left(y_{h^{0}}\right)-F_{1}\left(y_{A^{0}}\right)\right)\right.\right. \\
& \left.+2 \sum_{n=1}^{\infty} \frac{m_{\tau}}{\sqrt{m_{\tau}^{2}+m_{n}^{2}}}\left(F_{1}\left(y_{n, h^{0}}\right)-F_{1}\left(y_{n, A^{0}}\right)\right)\right)+m_{l_{1}} \bar{\xi}_{N, l_{2} \tau}^{E} \bar{\xi}_{N, l_{1} \tau}^{E *} c_{H}\left(\left(G\left(y_{h^{0}}\right)+G\left(y_{A^{0}}\right)\right)\right. \\
& \left.\left.+2 \sum_{n=1}^{\infty} \frac{m_{\tau}^{2}}{m_{\tau}^{2}+m_{n}^{2}}\left(G\left(y_{n, h^{0}}\right)+G\left(y_{n, A^{0}}\right)\right)\right)\right\},
\end{aligned}
$$

where $y_{n, S}=\frac{m_{\tau}^{2}+m_{n}^{2}}{m_{S}^{2}}, m_{n}=\frac{n}{R}$ and $Q_{\tau}$ is the charge of $\tau$ lepton. Here the vertex factor $c_{H}$ is defined in eq. (14). The function $F_{1}(w)$ is given in eq. (15) and $G(w)$ reads

$$
G(w)=\frac{w\left(2+3 w-6 w^{2}+w^{3}+6 w \ln w\right)}{(-1+w)^{4}} .
$$

\section{Discussion}

In this work, we study the EDMs of charged leptons and the BRs of the LFV $l_{1} \rightarrow l_{2} \gamma$ decays in the 2HDM with the addition of two spatial extra dimensions. Here we take that the leptons feel the fifth dimension, and the new Higgs doublet is localized with Gaussian profile in the sixth one. We consider that the extra dimensions are compactified on $S_{1} / Z_{2} \times S_{1}$ so that the chiralities of four dimensional lepton fields are guaranteed. On the other hand, we choose the location of the new Higgs doublet around the origin and also at the point near to the origin so that we estimate the possible effects coming from its position in the extra dimension.

The existence of the lepton EDM interactions depend on the CP violating phases and, in the present work, we consider the complex Yukawa couplings appearing in the FCNC at tree level in the framework of the 2HDM. The leptonic complex Yukawa couplings $\bar{\xi}_{N, i j}^{E}, i, j=e, \mu, \tau$ are set of free parameters in the 2HDM and we consider the Yukawa couplings $\bar{\xi}_{N, i j}^{E}, i, j=e, \mu$, as smaller compared to $\bar{\xi}_{N, \tau i}^{E} i=e, \mu, \tau$ and we assume that $\bar{\xi}_{N, i j}^{E}$ is symmetric with respect to the indices $i$ and $j$. Notice that the new Higgs masses are among the free parameters and we take their numerical values as $m_{h^{0}}=100 \mathrm{GeV}, m_{A^{0}}=200 \mathrm{GeV}$.

The inclusion of the spatial extra dimension that is felt by the leptons brings new contributions due to their KK excitations. The additional vertices appearing in the calculation of EDMs are coming from the fermion-KK fermion-new Higgs interaction where the KK number is not conserved. Here the compactification of fifth dimension results in a new parameter, called the compactification radius $R$, and it should take the numerical values not to contradict with the experimental measurements. For the new Higgs doublet, we consider the localization 
with Gaussian profile at any point near to the origin in the sixth dimension. Therefore, the localization width $\sigma=\rho R$ and the possible localization point $z_{H}=\alpha \sigma$ is other free parameters which should be examined. The direct limits from searching for KK gauge bosons imply $1 / R>800 \mathrm{GeV}$, the precision electro weak bounds on higher dimensional operators generated by KK exchange place a far more stringent limit $1 / R>3.0 \mathrm{TeV}$ [95] and, from $B \rightarrow \phi K_{S}$, the lower bounds for the scale $1 / R$ have been obtained as $1 / R>1.0 \mathrm{TeV}$, from $B \rightarrow \psi K_{S}$ one got $1 / R>500 \mathrm{GeV}$, and from the upper limit of the $B R, B R\left(B_{s} \rightarrow \mu^{+} \mu^{-}\right)<2.6 \times 10^{-6}$, the estimated limit was $1 / R>800 \mathrm{GeV}$ [80]. Here we take the compactification scale in the broad range, $100 \mathrm{GeV} \geq 1 / R \geq 1000 \mathrm{GeV}$, the width $\sigma=\rho R$ with $\rho \sim 0.001$ and the parameter $\alpha$, which regulates the localization point $z_{H}=\alpha \sigma$, in the interval $0.001 \geq \alpha \geq 1$.

Now, we start to estimate the charged lepton EDMs and to study the compactification scale $1 / R$ and the new Higgs location point dependencies of these measurable quantities.

In Fig. 2, we plot EDM $d_{e}$ with respect to the scale $1 / R$ for the intermediate value $\sin \theta_{e}=$ 0.5. Here the solid-dashed line (curve) represents the EDM for $\bar{\xi}_{N, \tau e}^{E}=0.001-0.01 \mathrm{GeV}$ without (with) lepton KK mode contribution in the case that the new Higgs doublet is located around the origin in the sixth dimension. The electron EDM is at the order of magnitude of $10^{-28}(e-\mathrm{cm})$ for the coupling $\bar{\xi}_{N, \tau e}^{E}=0.01 \mathrm{GeV}$ and enhances at the order of $25 \%$ for the compactification scale $1 / R \sim 500 \mathrm{GeV}$. We study the effect of the different location of the Gaussian profile of the new Higgs doublet on $d_{e}$ by plotting this quantity with respect to the $\alpha$ for $1 / R=1000 \mathrm{GeV}$ and $\bar{\xi}_{N, \tau e}^{E}=0.001 \mathrm{GeV}$ (see Fig. [3). Here the solid-dashed line (curve) represents the EDM without-with lepton KK mode contribution in the case that the new Higgs scalars are located around the origin $\left(z_{H}=\alpha \sigma\right)$ in the sixth dimension. We observe that the EDM is suppressed almost one order of magnitude even in the case that the Gaussian profiles are located one $\sigma$ farther from the origin. This shows that the EDM is strongly sensitive to the location of the Gaussian profiles of new Higgs scalars in the sixth dimension.

Fig. 4 is devoted to $\mathrm{EDM} d_{\mu}$ with respect to the scale $1 / R$ for the intermediate value $\sin \theta_{\mu}=0.5$. Here the solid-dashed line (curve) represents the EDM for $\bar{\xi}_{N, \tau \mu}^{E}=1-10 \mathrm{GeV}$ without (with) lepton KK mode contribution in the case that the new Higgs doublet is located around the origin in the sixth dimension. The muon EDM is at the order of magnitude of $10^{-22}(e-c m)$ for the coupling $\bar{\xi}_{N, \tau \mu}^{E}=10 \mathrm{GeV}$ and enhances nearly $30 \%$ for the compactification scale $1 / R \sim 500 \mathrm{GeV}$. For the small values of the compactification scale the enhancement is almost one order of magnitude. We represent the effect of the different location of the Gaussian profile of the new Higgs doublet on $d_{\mu}$ in Fig. 5, by taking $1 / R=1000 \mathrm{GeV}$ and 
$\bar{\xi}_{N, \tau \mu}^{E}=10 \mathrm{GeV}$. Here the solid-dashed line (curve) represents the EDM without-with lepton KK mode contribution in the case that the new Higgs scalars are located around the origin $\left(z_{H}=\alpha \sigma\right)$ in the sixth dimension. We observe that the EDM is strongly sensitive to the location of the Gaussian profiles of new Higgs scalars in the sixth dimension and its magnitude decreases almost one order of magnitude for the case that the Gaussian profiles are located at most $\sigma$ farther from the origin.

Finally, we make the same analysis for $\tau$ lepton EDM $d_{\tau}$. In Fig. 6 we present $d_{\tau}$ with respect to the scale $1 / R$ for the intermediate value $\sin \theta_{\mu}=0.5$. Here the solid-dashed line (curve) represents the EDM for $\bar{\xi}_{N, \tau \tau}^{E}=20-50 \mathrm{GeV}$ without (with) lepton KK mode contribution in the case that the new Higgs doublet is located around the origin in the sixth dimension. $d_{\tau}$ is at the order of magnitude of $10^{-20}(e-\mathrm{cm})$ for the coupling $\bar{\xi}_{N, \tau \tau}^{E}=50 \mathrm{GeV}$ and enhances more than $20 \%$ for the compactification scale $1 / R \sim 500 \mathrm{GeV}$. For the small values of the compactification scale the enhancement is almost one order of magnitude. The effect of the different locations of the Gaussian profile of the new Higgs doublet on $d_{\tau}$ is represented in Fig. 7. In this figure we take $1 / R=1000 \mathrm{GeV}$ and $\bar{\xi}_{N, \tau \tau}^{E}=50 \mathrm{GeV}$. Here the solid-dashed line (curve) represents the EDM without-with lepton KK mode contribution in the case that the new Higgs doublet is located around the origin $\left(z_{H}=\alpha \sigma\right)$ in the sixth dimension. We observe that the EDM is strongly sensitive to the location of new Higgs doublet Gaussian profiles in the sixth dimension and its magnitude is suppressed up to values less than one order of magnitude for the case that the Gaussian profile is located at most one $\sigma$ farther from the origin.

Now we continue our analysis for the BRs of the LFV decays $l_{1} \rightarrow l_{2} \gamma$. First, we consider that the new Higgs is localized around the origin in the extra dimension. Furthermore, we choose the localization point is near to the origin and study its effect on the BRs.

In Fig. 8, we plot $\mathrm{BR}(\mu \rightarrow e \gamma)$ with respect to the scale $1 / R$. Here the solid-dashed line (curve) represents the $\mathrm{BR}$ for $\bar{\xi}_{N, \tau e}^{E}=0.001 \mathrm{GeV}$ and $\bar{\xi}_{N, \tau \mu}^{E}=1 \mathrm{GeV}-\bar{\xi}_{N, \tau \mu}^{E}=10 \mathrm{GeV}$ without (with) lepton KK mode contribution in the case that the new Higgs doublet is located around the origin in the sixth dimension. The $\operatorname{BR}(\mu \rightarrow e \gamma)$ is at the order of magnitude of $10^{-11}$ for the coupling $\bar{\xi}_{N, \tau \mu}^{E}=10 \mathrm{GeV}$. The inclusion of internal lepton KK modes results in $50 \%$ enhancement in the $\mathrm{BR}$ for the compactification scale $1 / R \sim 500 \mathrm{GeV}$. This enhancement is more than one order of magnitude for the small values of the scale $1 / R$. Fig. 9 is devoted to the new Higgs doublet Gaussian profile location scale $\alpha$ dependence of the $\mathrm{BR}(\mu \rightarrow e \gamma)$ for $1 / R=1000 \mathrm{GeV}$ and $\bar{\xi}_{N, \tau e}^{E}=0.001 \mathrm{GeV}, \bar{\xi}_{N, \tau \mu}^{E}=10 \mathrm{GeV}$. Here the solid-dashed line (curve) represents the $\mathrm{BR}$ without-with lepton KK mode contribution in the case that the new Higgs 
is located around the origin $\left(z_{H}=\alpha \sigma\right)$ in the sixth dimension. We observe that the $\mathrm{BR}$ is strongly sensitive to the location of new Higgs scalar Gaussian profiles in the sixth dimension and it is suppressed almost two orders of magnitude even in the case that the Gaussian profile is located one $\sigma$ farther from the origin.

Fig. 10 is devoted to the $\mathrm{BR}(\tau \rightarrow e \gamma)$ with respect to the scale $1 / R$. Here the solid-dashed line (curve) represents the BR for $\bar{\xi}_{N, \tau \tau}^{E}=50 \mathrm{GeV}$ and $\bar{\xi}_{N, \tau e}^{E}=0.001 \mathrm{GeV}-\bar{\xi}_{N, \tau e}^{E}=0.01 \mathrm{GeV}$ without (with) lepton KK mode contribution in the case that the new Higgs is located around the origin in the sixth dimension. The $\mathrm{BR}(\tau \rightarrow e \gamma)$ is at the order of magnitude of $10^{-13}$ for the coupling $\bar{\xi}_{N, \tau e}^{E}=0.01 \mathrm{GeV}$. The additional contribution coming from the internal lepton KK modes causes $50 \%$ enhancement in the BR for the compactification scale $1 / R \sim 500 \mathrm{GeV}$ and the BR increases up to the values $10^{-11}$ for the small values of the scale $1 / R$. Fig. 11 represents the scale $\alpha$ dependence of the $\operatorname{BR}(\tau \rightarrow e \gamma)$ for $1 / R=1000 \mathrm{GeV}$ and $\bar{\xi}_{N, \tau e}^{E}=0.01 \mathrm{GeV}$, $\bar{\xi}_{N, \tau \tau}^{E}=50 \mathrm{GeV}$. Here the solid-dashed line (curve) represents the BR without-with lepton KK mode contribution in the case that the new Higgs is located around the origin $\left(z_{H}=\alpha \sigma\right)$ in the sixth dimension. We observe that the BR is suppressed almost two orders of magnitude for the case that the Gaussian profile is located one $\sigma$ farther from the origin, similar to the $\mu \rightarrow e \gamma$ decay.

In Fig. 12 we present the $\operatorname{BR}(\tau \rightarrow \mu \gamma)$ with respect to the scale $1 / R$. Here the solid-dashed line (curve) represents the BR for $\bar{\xi}_{N, \tau \tau}^{E}=50 \mathrm{GeV}$ and $\bar{\xi}_{N, \tau \mu}^{E}=1 \mathrm{GeV}-\bar{\xi}_{N, \tau \mu}^{E}=10 \mathrm{GeV}$ without (with) lepton KK mode contribution in the case that the new Higgs doublet is located around the origin in the sixth dimension. The $\mathrm{BR}(\tau \rightarrow \mu \gamma)$ is at the order of magnitude of $10^{-7}$ for the coupling $\bar{\xi}_{N, \tau \mu}^{E}=10 \mathrm{GeV}$. With the addition of the internal lepton KK mode contributions it enhances more than $50 \%$ for the compactification scale $1 / R \sim 500 \mathrm{GeV}$. Fig. 13 shows the scale $\alpha$ dependence of the $\operatorname{BR}(\tau \rightarrow \mu \gamma)$ for $1 / R=1000 \mathrm{GeV}$ and $\bar{\xi}_{N, \tau \mu}^{E}=10 \mathrm{GeV}, \bar{\xi}_{N, \tau \tau}^{E}=50 \mathrm{GeV}$. Here the solid-dashed line (curve) represents the BR without-with lepton KK mode contribution in the case that the new Higgs doublet is located around the origin $\left(z_{H}=\alpha \sigma\right)$ in the sixth dimension. We observe that the BR is suppressed more than one order of magnitude for the case that the Gaussian profile is located one $\sigma$ farther from the origin.

Now we would like to summarize our results:

- The inclusion of KK modes due to the fifth dimension results in enhancement in the electron (muon, tau) EDM at the order of $25 \%$ (30\%, 20\%) for the compactification scale $1 / R \sim 500 \mathrm{GeV}$. 
- Charged Lepton EDMs are strongly sensitive to the location of new Higgs doublet Gaussian profile in the sixth dimension and their magnitudes are suppressed almost one order of magnitude even in the case that the Gaussian profile is located one $\sigma$ farther from the origin.

- The BRs of LFV $l_{1} \rightarrow l_{2} \gamma$ decays enhance almost $50 \%$ with the inclusion of KK modes due to the fifth dimension for the compactification scale $1 / R \sim 500 \mathrm{GeV}$. This enhancement is the almost two orders of magnitude for the small values of scale $1 / R$.

- The BRs of LFV $l_{1} \rightarrow l_{2} \gamma$ decays are strongly sensitive to the location of new Higgs doublet Gaussian profile in the sixth dimension and their magnitudes are suppressed almost two orders of magnitude even in the case that the Gaussian profile is located one $\sigma$ farther from the origin.

With the help of the forthcoming most accurate experimental measurements, the valuable information can be obtained about the existence of extra dimensions and the possibilities of Gaussian profiles of the Higgs scalars.

\section{References}

[1] C. R. Schmidt and M. E. Peskin, Phys. Rev. Lett. 69, 410 (1992).

[2] E. D. Commins et.al. Phys. Rev. A 50, 2960 (1994).

[3] J. Bailey et al, Journ. Phys. G4 (1978) 345.

[4] Particle Data Group, D. E. Groom et.al. European Phys. Journ. C15 (2000) 1.

[5] B. Dutta, R. N. Mohapatra, Phys. Rev. D68, 113008 (2003).

[6] E. Iltan, Phys. Rev. D64, 013013 (2001).

[7] E. Iltan, JHEP 065, 0305 (2003).

[8] E. Iltan, JHEP 0404, 018 (2004).

[9] E. Iltan, Eur. Phys. C44, 411 (2005).

[10] E. Iltan, hep-ph/0511241.

[11] M. L. Brooks et. al., MEGA Collaboration, Phys. Rev. Lett. 83, 1521 (1999). 
[12] K. Hayasaka et al.., Phys. Lett. B613, 20 (2005).

[13] Donato Nicolo, MUEGAMMA Collaboration, Nucl. Instrum. Meth A503, 287 (2003).

[14] S. Yamada, Nucl. Phys. Proc. Suppl. 144, 185 (2005).

[15] J.M. Roney and the BABAR Collaboration, Nucl. Phys. Proc. Suppl. 144, 155 (2005).

[16] B. Aubert et. al., BABAR Collaboration, SLAC-PUB-11028, BABAR-PUB-04-049, Feb. 2005, 7. pp, Phys. Rev. Lett. 95, 041802 (2005).

[17] R. Barbieri and L. J. Hall, Phys. Lett. B338, 212 (1994).

[18] R. Barbieri, L. J. Hall and A. Strumia, Nucl. Phys. B445, 219 (1995).

[19] R. Barbieri, L. J. Hall and A. Strumia, Nucl. Phys. B449, 437 (1995).

[20] P. Ciafaloni, A. Romanino and A. Strumia, IFUP-YH-42-95.

[21] T. V. Duong, B. Dutta and E. Keith, Phys. Lett. B378, 128 (1996).

[22] G. Couture, et. al., Eur. Phys. J. C7, 135 (1999).

[23] Y. Okada, K. Okumara and Y. Shimizu, Phys. Rev. D61, 094001 (2000).

[24] D. Chang, W. S. Hou and W. Y. Keung, Phys. Rev. D48, 217 (1993).

[25] E. O. Iltan, Phys. Rev. D64, 115005 (2001).

[26] E. O. Iltan, Phys. Rev. D64, 013013 (2001).

[27] R. Diaz, R. Martinez and J-Alexis Rodriguez, Phys. Rev. D63, 095007 (2001).

[28] E. O. Iltan, JHEP 0408, 20 (2004).

[29] E. O. Iltan, hep-ph/0504013, (2005).

[30] E. O. Iltan, Eur.Phys.J. C46, 475 (2006).

[31] E. O. Iltan, hep-ph/0602170, (2006).

[32] P. Paradisi, JHEP 0602, 050 (2006).

[33] N. Arkani-Hamed, S. Dimopoulos, and G. R. Dvali, Phys. Rev. D59, 086004 (1999). 
[34] N. Arkani-Hamed, S. Dimopoulos, and G. R. Dvali, Phys. Lett. B429, 263 (1998).

[35] I. Antoniadis, Phys. Lett. B246, 377 (1990).

[36] I.Antoniadis and K. Benakli, Phys. Lett. B326, 69 (1994).

[37] I. Antoniadis, K. Benakli, and M. Quiros, Phys. Lett. B331, 313 (1994).

[38] A. Pomarol and M. Quiros, Phys. Lett. B438, 255 (1998).

[39] I. Antoniadis, K. Benakli, and M. Quiros, Phys. Lett. B460, 176 (1999).

[40] P. Nath, Y. Yamada, and M. Yamaguchi, Phys. Lett. B466, 100 (1999).

[41] M. Masip and A. Pomarol, Phys. Rev. D60, 096005 (1999).

[42] A. Delgado, A. Pomarol, and M. Quiros, JHEP 01, 030 (2000).

[43] T. G. Rizzo and J. D. Wells, Phys. Rev. D61, 016007 (2000).

[44] P. Nath and M. Yamaguchi, Phys. Rev. D60, 116004 (1999).

[45] A. Muck, A. Pilaftsis, and R. Ruckl, Phys. Rev. D65, 085037 (2002).

[46] A. Muck, A. Pilaftsis, and R. Ruckl, hep-ph/0203032 (2002).

[47] C. D. Carone, Phys. Rev. D61, 015008 (2000).

[48] I. Antoniadis, C. Munoz, M. Quiros, Nucl. Phys B397, 515 (1993).

[49] T. Appelquist, H.-C. Cheng, B. A. Dobrescu, Phys. Rev. D64, 035002 (2001).

[50] J. Papavassiliou and A. Santamaria, Phys. Rev. D63, 016002 (2001).

[51] D. Chakraverty, K. Huitu, and A. Kundu, Phys. Lett. B558, 173 (2003).

[52] A. J. Buras, M. Spranger, and A. Weiler, Nucl. Phys. B660, 225 (2003).

[53] K. Agashe, N.G. Deshpande, G.-H. Wu, Phys. Lett. 514, 309 (2001).

[54] K. R. Dienes, E. Dudas, T. Gherghetta, Nucl. Phys. B557, 25 (1999).

[55] Q. H. Cao, S. Gopalakrishna, C. P. Yuan, Phys. Rev. D69, 115003 (2004).

[56] F. Agulia, M. P. Victoria, J. Santiago, JHEP 0302 051, (2003). 
[57] F. Agulia, M. P. Victoria, J. Santiago, Acta Phys.Polon. B34, 5511 (2003).

[58] E. O. Iltan, Phys. Scripta 73, 531 (2006).

[59] E. O. Iltan, JHEP 0402, 065 (2004).

[60] E. O. Iltan, Mod. Phys. Lett. A20, 1845 (2005).

[61] E. O. Iltan, Eur. Phys. J. C41, 233 (2005).

[62] C. S. Lam, hep-ph/0302227, (2003).

[63] C. A. Scrucca, M. Serona, L. Silvestrini, Nucl.Phys. B669 128, (2003).

[64] M. Gozdz, W. A. Kaminsk, Phys. Rev. D68, 057901 (2003).

[65] C. Biggio, et.al, Nucl.Phys. B677, 451 (2004).

[66] M. Carena, et.al, Phys. Rev. D68, 035010 (2003).

[67] A. J. Buras, et. al., Nucl.Phys. B678, 455 (2004).

[68] T. G. Rizzo, JHEP 0308, 051 (2003),

[69] A. J. Buras, hep-ph/0307202, (2003).

[70] S. Matsuda, S. Seki, hep-ph/0307361, (2003).

[71] R. N. Mohapatra, Phys. Rev. D68, 116001, (2003).

[72] B. Lillie, JHEP 0312, 030 (2003).

[73] A.A. Arkhipov, hep-ph/0309327 (2003).

[74] N. Haba, JHEP 0605, 030 (2006).

[75] F.Feruglia, Eur.Phys.J. C33, 114 (2004).

[76] E. A. Mirabelli, Schmaltz, Phys. Rev. D61, 113011 (2000).

[77] W. F. Chang, I. L. Ho and J. N. Ng, Phys. Rev. D66, 076004 (2002).

[78] G. C. Branco,A. Gouvea, M. N. Rebelo, Phys. Lett. B506, 115 (2001).

[79] W. F. Cang, J. N. Ng, JHEP 0212, 077 (2002). 
[80] B. Lillie, J. L. Hewett, Phys. Rev. 68, 116002 (2003).

[81] Y. Grossman and G. Perez, Phys. Rev. D67, 015011 (2003).

[82] Y. Grossman and G. Perez, Pramana 62, 733 (2004).

[83] Y. Grossman, Int. J. Mod. Phys. A15, 2419 (2000).

[84] D. E. Kaplan and T. M. Tait, JHEP 0111, 051 (2001).

[85] G. Barenboim, et. al., Phys. Rev. D64, 073005 (2001).

[86] W. F. Chang, I. L. Ho and J. N. Ng, Phys. Rev. D66, 076004 (2002).

[87] W. Skiba and D. Smith, Phys. Rev. D65, 095002 (2002).

[88] Y. Grossman, R. Harnik, G. Perez, M. D. Schwartz and Z. Surujon, Phys. Rev. D71, 056007 (2005).

[89] P. Dey, G. Bhattacharya, Phys. Rev. D70, 116012 (2004).

[90] Y. Nagatani, G. Perez, JHEP 0502, 068 (2005).

[91] R. Harnik, G. Perez, M. D. Schwartz, Y. Shirman, JHEP 0503, 068 (2005).

[92] Z. Surujon, Phys.Rev. D73, 016008 (2006).

[93] E. O. Iltan, hep-ph/0603192, (2006).

[94] T. P. Cheng and M. Sher, Phy. Rev. D35, 3383 (1987).

[95] T. G. Rizzo, J. D. Wells, Phy. Rev. D61, 016007 (2000). 


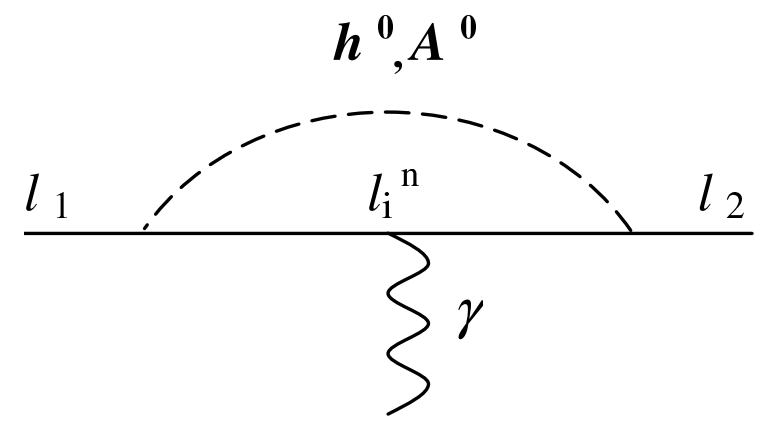

Figure 1: One loop diagrams contribute to $l_{1} \rightarrow l_{2} \gamma$ decay due to the zero mode neutral Higgs bosons $h^{0}$ and $A^{0}$ in the 2HDM, for a single extra dimension. These diagrams contribute to EDM of charged lepton $l_{1}$ for $l_{1}=l_{2}$. Here $l_{i}^{(n)}$ represents the internal KK mode charged lepton and $n=0,1, \ldots$ Wavy lines represent the electromagnetic field, dashed line the Higgs field, solid line the charged leptons $l_{1(i)}=e, \mu, \tau$. 


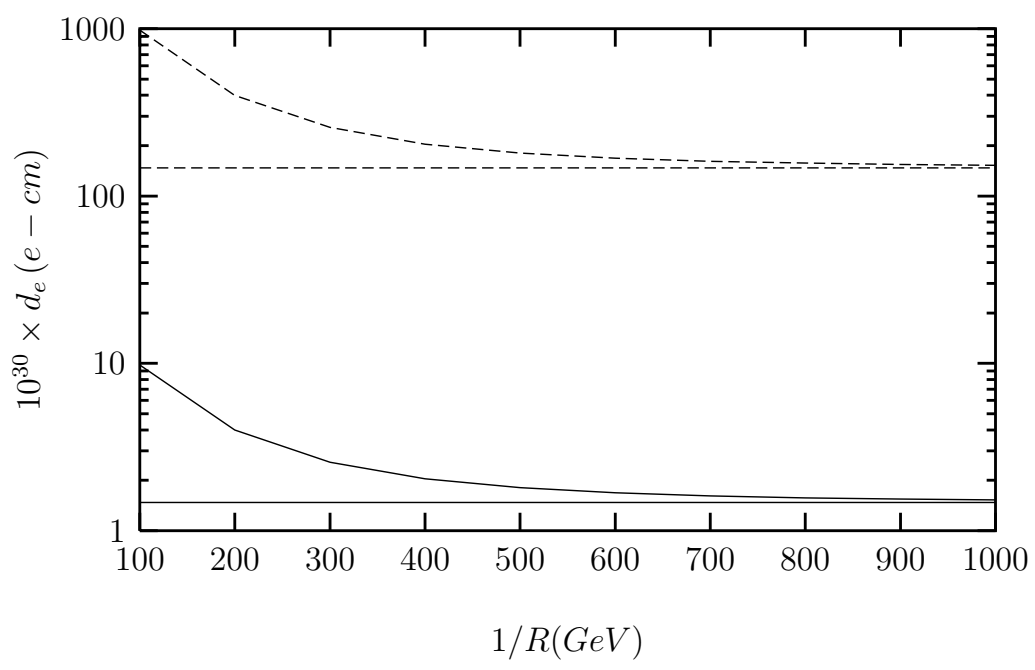

Figure 2: $d_{e}$ with respect to $1 / R$ and $\sin \theta_{e}=0.5$. Here the solid-dashed line (curve) represents the EDM for $\bar{\xi}_{N, \tau e}^{E}=0.001-0.01 \mathrm{GeV}$ without (with) lepton KK mode contribution in the case that the new Higgs doublet is located around the origin in the sixth dimension.

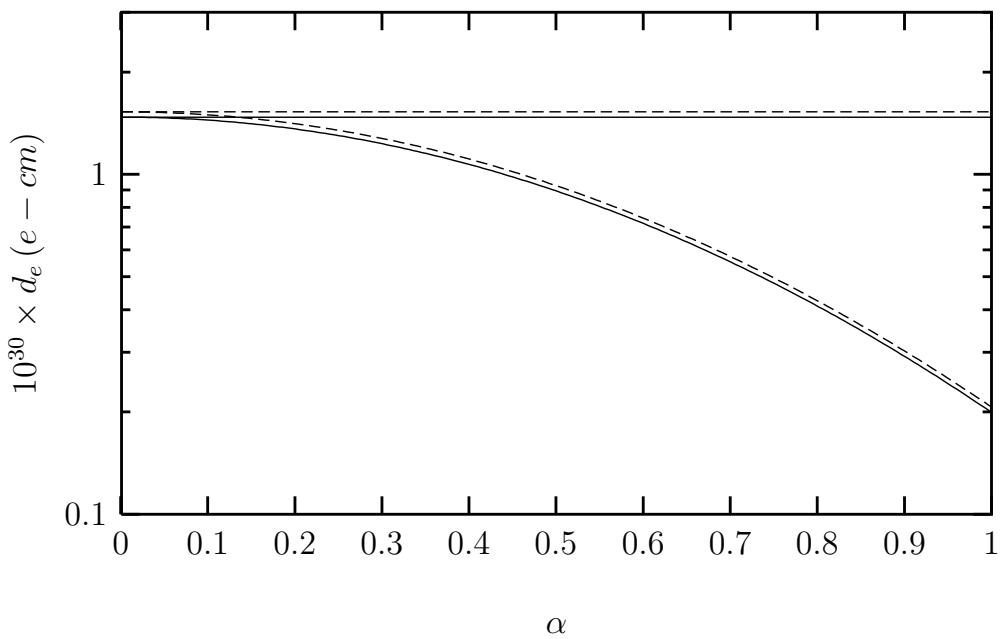

Figure 3: $d_{e}$ with respect to $\alpha$ for $1 / R=1000 \mathrm{GeV}$ and $\bar{\xi}_{N, \tau e}^{E}=0.001 \mathrm{GeV}$. Here the soliddashed line (curve) represents the EDM without-with lepton KK mode contribution in the case that the new Higgs scalars are located around the origin $\left(z_{H}=\alpha \sigma\right)$ in the sixth dimension. 


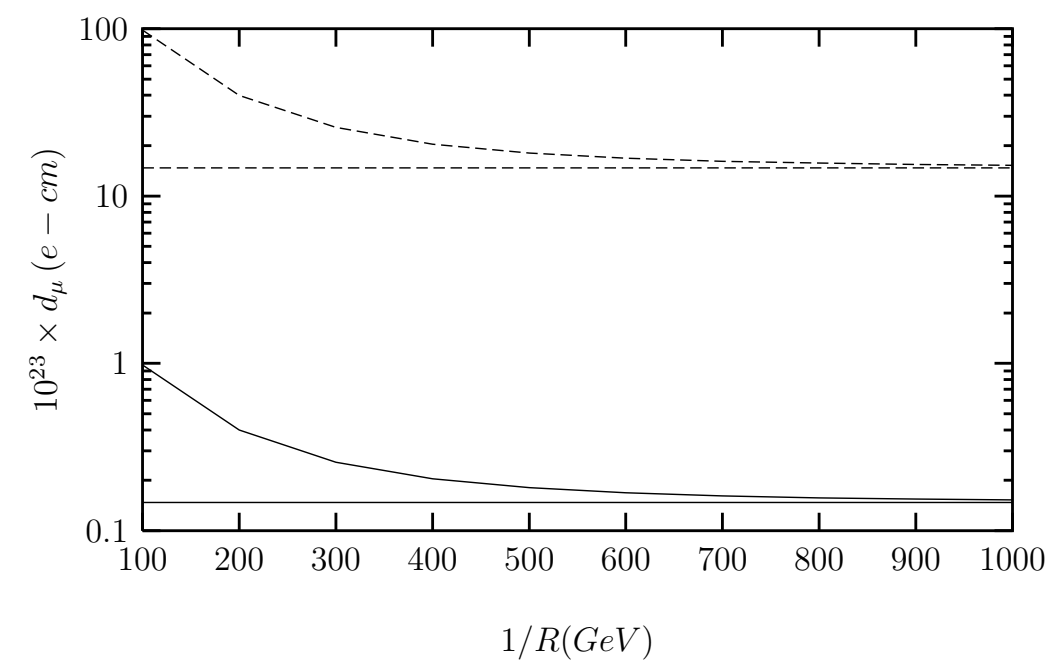

Figure 4: $d_{\mu}$ with respect to $1 / R$ for $\sin \theta_{\mu}=0.5$. Here the solid-dashed line (curve) represents the EDM for $\bar{\xi}_{N, \tau \mu}^{E}=1-10 \mathrm{GeV}$ without (with) lepton KK mode contribution in the case that the new Higgs doublet is located around the origin in the sixth dimension.

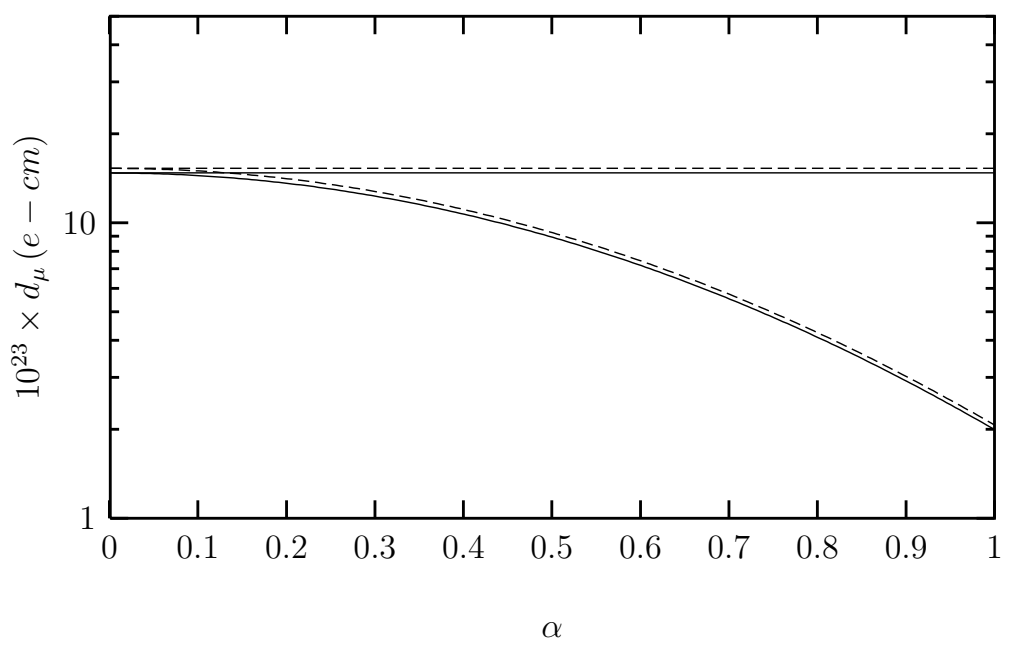

Figure 5: $d_{\mu}$ with respect to $\alpha$ for $1 / R=1000 \mathrm{GeV}$ and $\bar{\xi}_{N, \tau \mu}^{E}=10 \mathrm{GeV}$. Here the solid-dashed line (curve) represents the EDM without-with lepton KK mode contribution in the case that the new Higgs scalars are located around the origin $\left(z_{H}=\alpha \sigma\right)$ in the sixth dimension. 


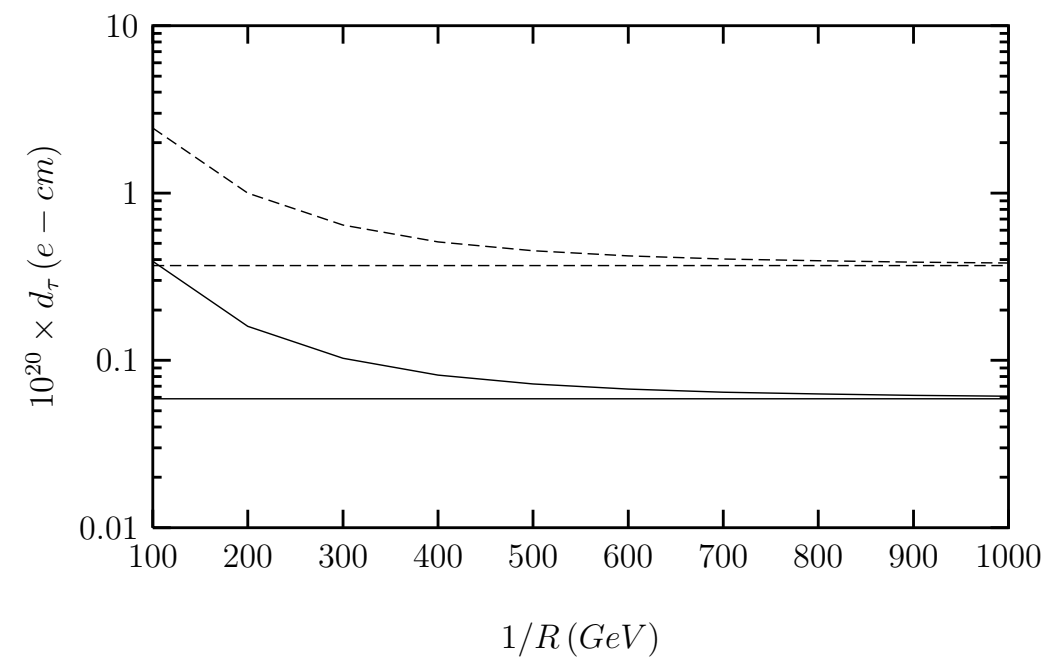

Figure 6: $d_{\tau}$ with respect to $1 / R$ for $\sin \theta_{\tau}=0.5$. Here the solid-dashed line (curve) represents the EDM for $\bar{\xi}_{N, \tau \tau}^{E}=20-50 \mathrm{GeV}$ without (with) lepton KK mode contribution in the case that the new Higgs doublet is located around the origin in the sixth dimension.

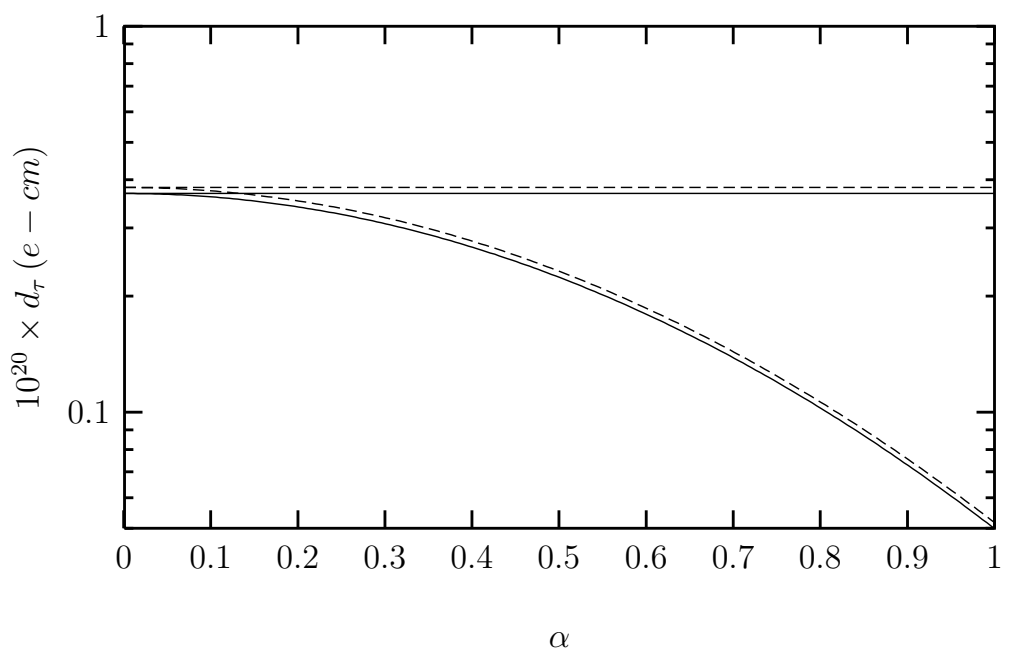

Figure 7: $d_{\tau}$ with respect to $\alpha$ for $1 / R=1000 \mathrm{GeV}$ and $\bar{\xi}_{N, \tau \tau}^{E}=50 \mathrm{GeV}$. Here the solid-dashed line (curve) represents the EDM without-with lepton KK mode contribution in the case that the new Higgs scalars are located around the origin $\left(z_{H}=\alpha \sigma\right)$ in the sixth dimension. 


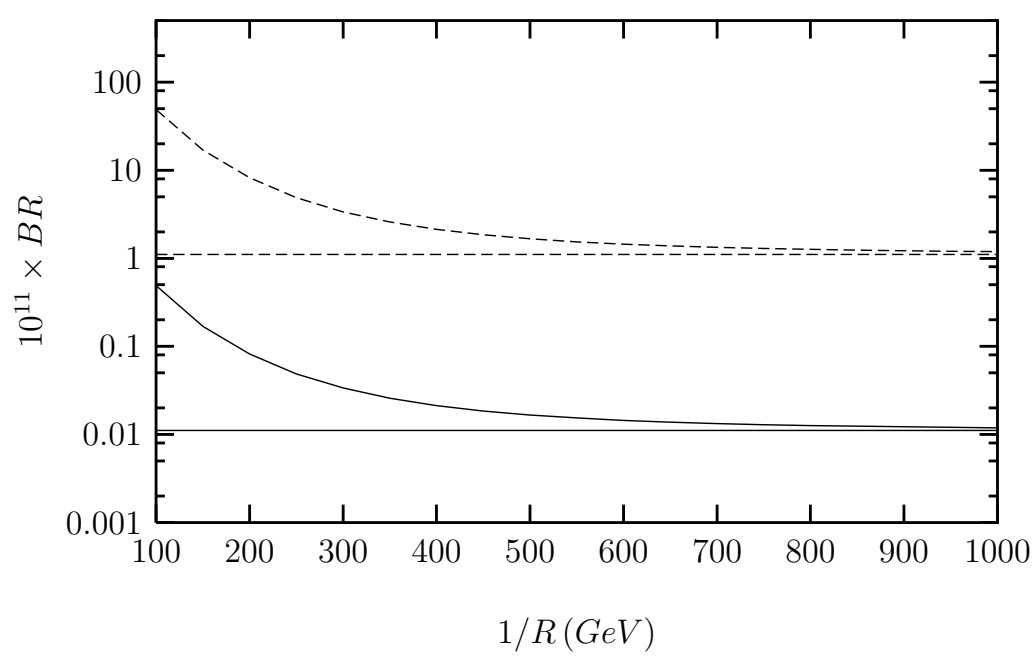

Figure 8: $\mathrm{BR}(\mu \rightarrow e \gamma)$ with respect to $1 / R$. Here the solid-dashed line (curve) represents the BR for $\bar{\xi}_{N, \tau e}^{E}=0.001 \mathrm{GeV}$ and $\bar{\xi}_{N, \tau \mu}^{E}=1 \mathrm{GeV}-\bar{\xi}_{N, \tau \mu}^{E}=10 \mathrm{GeV}$ without (with) lepton KK mode contribution in the case that the new Higgs doublet is located around the origin in the sixth dimension.

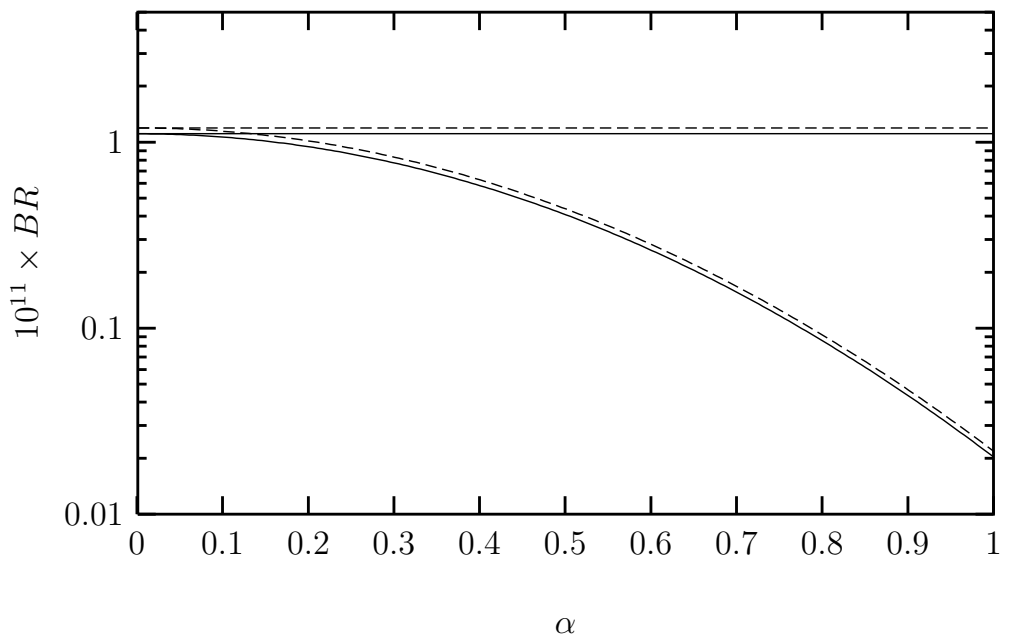

Figure 9: $\operatorname{BR}(\mu \rightarrow e \gamma)$ with respect to $\alpha$ for $1 / R=1000 \mathrm{GeV}$ and $\bar{\xi}_{N, \tau e}^{E}=0.001 \mathrm{GeV}, \bar{\xi}_{N, \tau \mu}^{E}=$ $10 \mathrm{GeV}$. Here the solid-dashed line (curve) represents the BR without-with lepton KK mode contribution in the case that the new Higgs doublet is located around the origin $\left(z_{H}=\alpha \sigma\right)$ in the sixth dimension. 


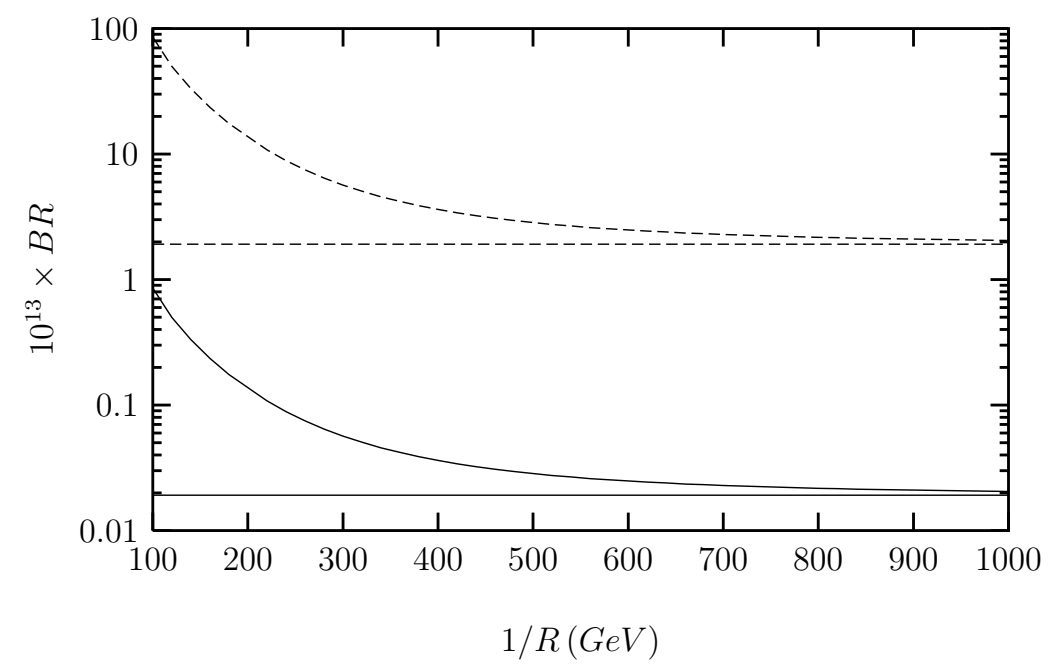

Figure 10: $\mathrm{BR}(\tau \rightarrow e \gamma)$ with respect to $1 / R$. Here the solid-dashed line (curve) represents the $\mathrm{BR}$ for $\bar{\xi}_{N, \tau \tau}^{E}=50 \mathrm{GeV}$ and $\bar{\xi}_{N, \tau e}^{E}=0.001 \mathrm{GeV}-\bar{\xi}_{N, \tau e}^{E}=0.01 \mathrm{GeV}$ without (with) lepton KK mode contribution in the case that the new Higgs doublet is located around the origin in the sixth dimension.

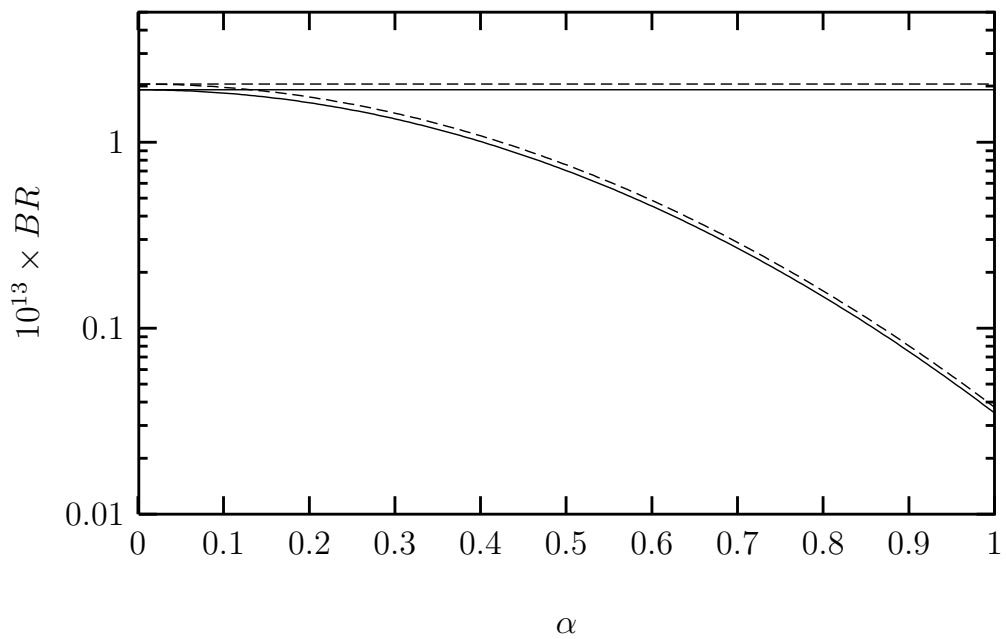

Figure 11: $\operatorname{BR}(\tau \rightarrow e \gamma)$ with respect to $\alpha$ for $1 / R=1000 \mathrm{GeV}$ and $\bar{\xi}_{N, \tau e}^{E}=0.01 \mathrm{GeV}, \bar{\xi}_{N, \tau \tau}^{E}=$ $50 \mathrm{GeV}$. Here the solid-dashed line (curve) represents the BR without-with lepton KK mode contribution in the case that the new Higgs doublet is located around the origin $\left(z_{H}=\alpha \sigma\right)$ in the sixth dimension. 


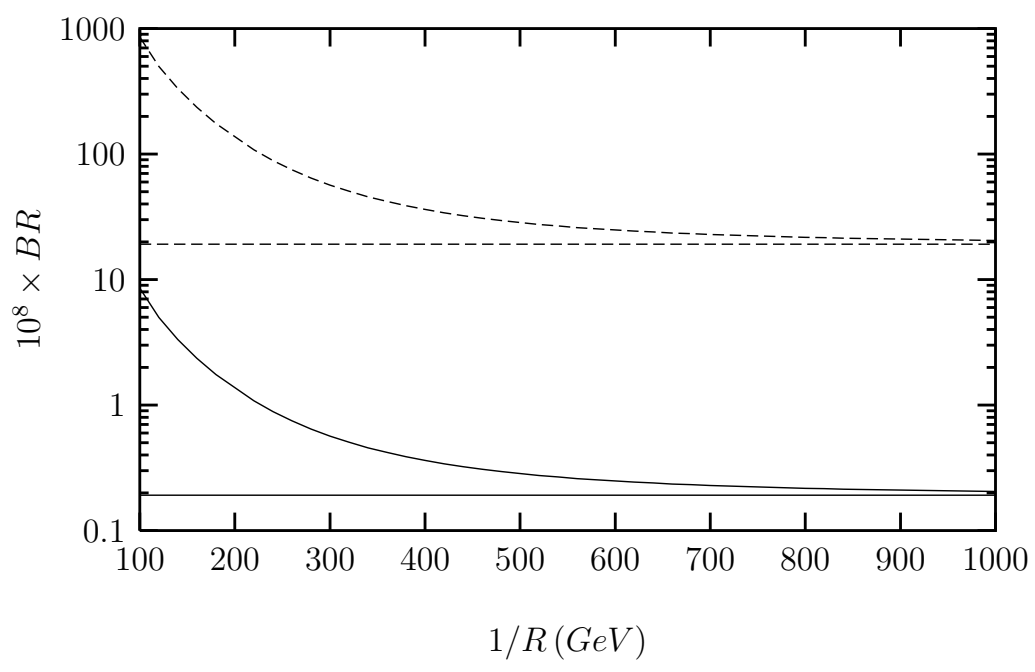

Figure 12: $\operatorname{BR}(\tau \rightarrow \mu \gamma)$ with respect to $1 / R$. Here the solid-dashed line (curve) represents the BR for $\bar{\xi}_{N, \tau \tau}^{E}=50 \mathrm{GeV}$ and $\bar{\xi}_{N, \tau \mu}^{E}=1 \mathrm{GeV}-\bar{\xi}_{N, \tau \mu}^{E}=10 \mathrm{GeV}$ without (with) lepton KK mode contribution in the case that the new Higgs doublet is located around the origin in the sixth dimension.

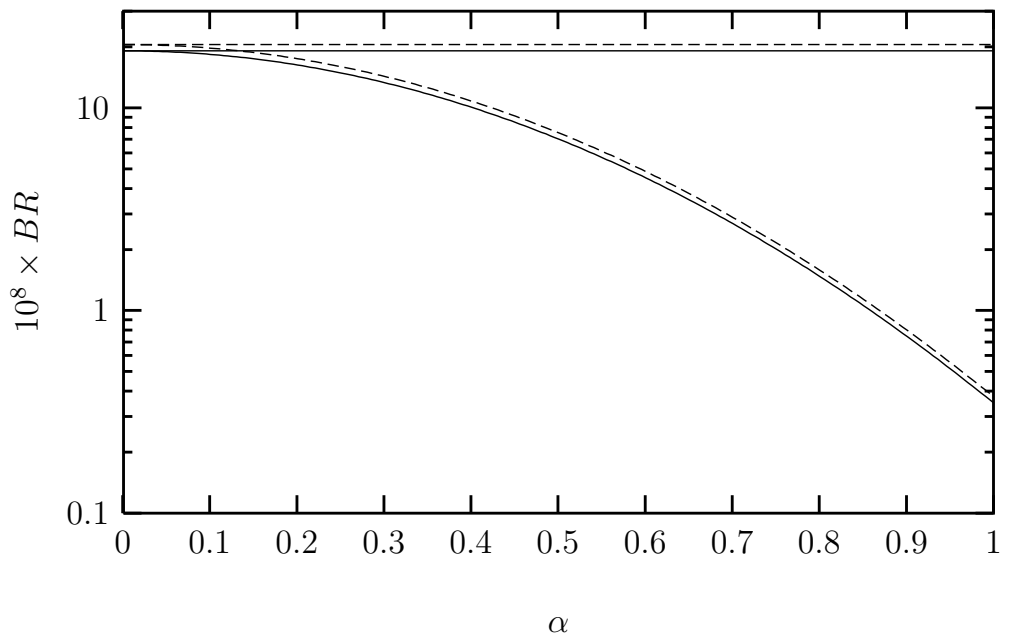

Figure 13: $\operatorname{BR}(\tau \rightarrow \mu \gamma)$ with respect to $\alpha$ for $1 / R=1000 \mathrm{GeV}$ and $\bar{\xi}_{N, \tau \mu}^{E}=10 \mathrm{GeV}, \bar{\xi}_{N, \tau \tau}^{E}=$ $50 \mathrm{GeV}$. Here the solid-dashed line (curve) represents the BR without-with lepton KK mode contribution in the case that the new Higgs doublet is located around the origin $\left(z_{H}=\alpha \sigma\right)$ in the sixth dimension. 